\title{
THERMAL AND FLAME RETARDANT PROPERTIES OF SHAPED POLYPROPYLENE FIBERS CONTAINING MODIFIED-THAI BENTONITE
}

\author{
Chureerat Prahsarn $^{1 *}$, Nanjaporn Roungpaisan², Wattana Klinsukhon ${ }^{1}$, Natthaphop Suwannamek ${ }^{1}$, Sirada Padee ${ }^{1}$ \\ ${ }^{1}$ National Metal and Materials Technology Center, 114 Pathoyothin Klong 1, Klong Luang, Pathumthani 12120, Thailand. \\ Phone 6625646500 Fax 6625646446 \\ 2Department of Engineering, Ratchamangkala University of Technology, 39 Moo 1, Rangsit-Nakhonnayok Rd., Thanyaburi, Pathumthani 12110, Thailand. \\ *Email chureerp@mtec.or.th
}

\begin{abstract}
:
Tetraphenyl phosphonium-modified organoclay (TPP-Mt) was prepared by modifying montmorillonite-rich Thai bentonite via ion exchange. TGA results revealed that TPP-Mt possessed high thermal stability, where degradation occurred at a temperature range of $418-576^{\circ} \mathrm{C}$. The obtained TPP-Mt/PP nanocomposites exhibited degradation at higher temperatures than $\mathrm{PP}\left(410-420^{\circ} \mathrm{C}\right.$ vs. $\left.403^{\circ} \mathrm{C}\right)$. Fibers of different cross-sectional shapes (circular, circular hollow, and cross) containing 1, 2 and 3\%wt TPP-Mt were prepared and characterized. Nonwovens of 3\%wt TPPMt/PP fibers were fabricated for flame retardant test. From results, nonwovens of TPP-Mt/PP fibers exhibited selfextinguishing characteristic and the areas of burning were less than that of PP nonwoven (14.5-31.6\% vs. 95.6\%). Nonwovens of cross-shaped fibers showed the best flame retardant property, followed by those of circular hollow and circular fibers. The flame retardant properties observed in nonwovens were explained due to the inter-fiber spaces between cross-shaped fibers and center hole in circular hollow fibers, which could trap initiating radicals inside, thus reducing flame propagation. In addition, large surface area in cross-shaped fibers could help in increasing the flame retardant effectiveness due to more exposure of TPP-Mt particles to the flame. Knowledge obtained in this study offered an approach to produce flame retardant nonwovens via a combination of modified organolcay and fiber shape.
\end{abstract}

\section{Keywords:}

Modified organoclay, Flame retardant, Shaped fibers, Nonwovens

\section{Introduction}

Organoclay-polymer nanocomposites have received great attention for decades as for their enhanced mechanical, thermal, and flame retardant properties, achieved by the addition of a small amount of organoclay. ${ }^{[1-15]}$ The properties of organoclay-polymer nanocomposites are governed by the dispersity of organoclay in polymer matrix and degree of polymer intercalation. Highly exfoliated organoclay with large basal spacing tends to allow effective mixing with polymer matrix, thus improved properties. In practice, hydrophilic montmorillonite-rich clay is modified with organic modifiers via ion exchange. The exchangeable cations locating between clay interlayers are exchanged with bulky organic ions of the modifiers. The exchanged organic ions tend to increase basal spacing between the clay layers due to their steric complexity. The monomer or polymer chains can enter into clay interlayers and cause further expansion, thus better mixing between clays and polymer matrix. ${ }^{1-2]}$

Organic modifiers such as phosphonium, imidazolium, and Pyridium salts had been employed to modify montmorillonite-rich clay to obtain organoclay with high thermal stability. Calderon et al. ${ }^{[6]}$ reported high thermal stability in alkyl phosphoniummodified montmorillonites that 5 percent mass loss occurred at a temperature range of $304-405^{\circ} \mathrm{C}$, which was higher than those of typical ammonium-modified montmorillonites (233 and $287^{\circ} \mathrm{C}$ ). The extent of thermal improvement depended on structures of the phosphonium ions. Abdallah and Yilmazer ${ }^{[7]}$ prepared high thermal stable organo-montmorillonites via ion exchange of sodium cations in montmorillonite-rich Turkish bentonites with different organic surfactants: (alkyl, aryl) phosphonium and di-(alkyl, aryl) imidazolium. Results showed that the onset decomposition temperatures of the obtained organo-montmorillonites occurred at $327-437^{\circ} \mathrm{C}$, which were considerably thermal stable for polymer processing of $200-350^{\circ} \mathrm{C}$. Similarly, Mittal ${ }^{[8]}$ studied the thermal stability of montmorillonites modified with surfactants of phosphonium, imidazolium, and pyridium ions. TGA results revealed that phosphonium-modified montmorillonite showed maximum thermal stability with degradation at $600^{\circ} \mathrm{C}$, and the time to maintain 1 percent mass loss was longer than 200 minutes.

In textiles for special use, such as home textiles, protective clothing and wall panel, flame retardant performance is required. Melt spinning of organoclay-polymer nanocomposites enables permanent flame retardant property in the fibers. Studies on organoclay-polymer nanocomposites have been conducted extensively in films and composites, however, very few was conducted in fibers. ${ }^{[11-15]}$ Shaped fibers possessed noncircular cross-sectional profiles such as circular hollow, flat, triangular, star, and cross. Their non-circular cross-sections 
provide special features and functions such as softness, luster, resilience, moisture transport, and bending stiffness. ${ }^{[16-22]}$ Melt spinning of shaped fibers was complex, and to the best of our knowledge, the melt spinning of organoclay-polymer shaped fibers, as well as the influence of fiber shape on thermal and flame retardant properties, is yet to be explored.

In this study, montmorillonite-rich Thai bentonite was modified with phosphonium salt and added into polypropylene to prepare nanocomposite. Three shaped fibers of phosphonium-modified montmorillonite/polypropylene (TPP-Mt/PP) nanocomposite were prepared by melt spinning, and characterized for their thermal properties. Flame retardant properties of their dry-laid nonwovens were also investigated to explore the influence of modified montmorillonite and the fibers' cross-sectional shapes.

\section{Experimental}

\subsection{Materials}

Montmorillonite-rich bentonite, supported by Thai Nippon Co. Ltd., was further purified as described elsewhere. ${ }^{[23-24]}$ In summary, bentonite was dispersed in deionized water (ratio 5g: 1L) and stirred for $24 \mathrm{~h}$, followed by sonication for $20 \mathrm{~min}$. The bentonite slurry was then left to settle for $50 \mathrm{~h}$. The upper fraction of slurry with dispersed clay particles was collected and slowly oven dried at $90^{\circ} \mathrm{C}$. Tetraphenyl phosphonium bromide salt (TPP-Br) purchased from Aldrich were used as received. Polypropylene (MFI = $25 \mathrm{~g} / 10 \mathrm{~min}$, density $0.90 \mathrm{~g} / \mathrm{cm}^{3}$ ) obtained from HMC Polymers (Thailand) was used as received.

\subsection{Preparation of tetraphenyl phosphonium-modified montmorillonite (TPP-Mt)}

To prepare TPP-Mt, purified bentonite (Mt) was dispersed in deionized water (at ratio $5 \mathrm{~g}: 1 \mathrm{~L}$ ) and continuously stirred for $30 \mathrm{~min}$, using a disperser (Ultra-Turrax® T50, IKA). An equal amount of phosphonium salt was then slowly added to the bentonite slurry and stirred vigorously at high speed ( 6000 rpm) for $30 \mathrm{~min}$ to allow intercalation of tetraphenyl phosphonium into Mt via ion exchange, before further stirring at medium speed (3000 rpm) for another $3 \mathrm{~h}$. The TPP-Mt was filtered and washed with deionized water until no bromide residual was left and then dried at $110^{\circ} \mathrm{C}$ for 24 hours. The thermal properties of purified Mt and TPP-Mt were characterized by thermogravimetric analysis
(Mettler Toledo, SDTA $851^{\circ}$ ). The samples were heated to $1200^{\circ} \mathrm{C}$ with heating rate $10^{\circ} \mathrm{C} / \mathrm{min}$ under $\mathrm{N}_{2}$ flow of $20 \mathrm{ml} / \mathrm{min}$. The structures of Mt and TPP-Mt were characterized, using WAXD (JEOL, JDX3530) with CuKa source. To prepare XRD sample, the fibers were arranged in parallel to one another on the sample holder. In the experiment, the intensity distributions

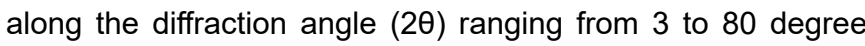
scanning rate $0.02 \mathrm{deg} / \mathrm{min}$ were measured.

\subsection{Preparation of TPP-MT/PP shaped fibers}

The 20\%wt TPP-Mt/PP masterbatch was first prepared via compounding at $200^{\circ} \mathrm{C}$ and screw speed of $80 \mathrm{rpm}$, using twin screw extruder. The obtained masterbatch and virgin PP were dried under vacuum at $80^{\circ} \mathrm{C}$ for $12 \mathrm{~h}$, and then mixed to yield the composition ratios of 1,2 , and $3 \%$ wt TPP-Mt. In fiber spinning, (1-3\%wt) TPP-Mt/PP mixtures were melt spun at $200^{\circ} \mathrm{C}$ through a spinneret of different cross-sectional shapes (circular, circular hollow, and cross) and taken up at a speed of $100 \mathrm{~m} / \mathrm{min}$ to obtain TPP-Mt/PP fibers, in comparison with PP. The profiles and dimensions of spinneret's holes are shown in Fig. 1. The quenching air temperature was $20^{\circ} \mathrm{C}$, with flow speed of 0.3-0.4 m/s. The cross-sectional shape and perimeter of the obtained PP and TPP-Mt/PP fibers were investigated and characterized using an optical microscope (National Optical \& Scientific Instrument, Inc.) and SEM (JEOL, JSM-6301F). In measurement, fiber bundle was placed in narrow slit of sample holder and cross-sectioned. Images of fiber cross-sectional features, observed under OM, were recorded and analyzed for their perimeters, using ImageJ software.

\subsection{Nonwovens of TPP-Mt/PP fibers and their flame retardant properties}

Dry-laid nonwovens of 5-mm thickness and $1,000 \mathrm{~g} / \mathrm{m}^{2}$ in weight were prepared from the $3 \%$ wt TPP-Mt/PP fibers and PP fibers for flame retardant test (ASTM D1230: Flame Resistance of Textile), using Flammability speed tester (DAIEI KAGAKU SEIKI MFG. CO., LTD). In testing, nonwoven sample was fixed in sample slot and set at 45-degree angle to flame source. The sample was held in touch with the flame for 60 seconds before the fire source was removed. Flame propagation on nonwoven sample were recorded at time 0 (first touch), 1, 15, 30, 45 and 60 seconds. Five replicas of nonwoven samples were tested. After each test, the area of burning was determined, using ImageJ software.
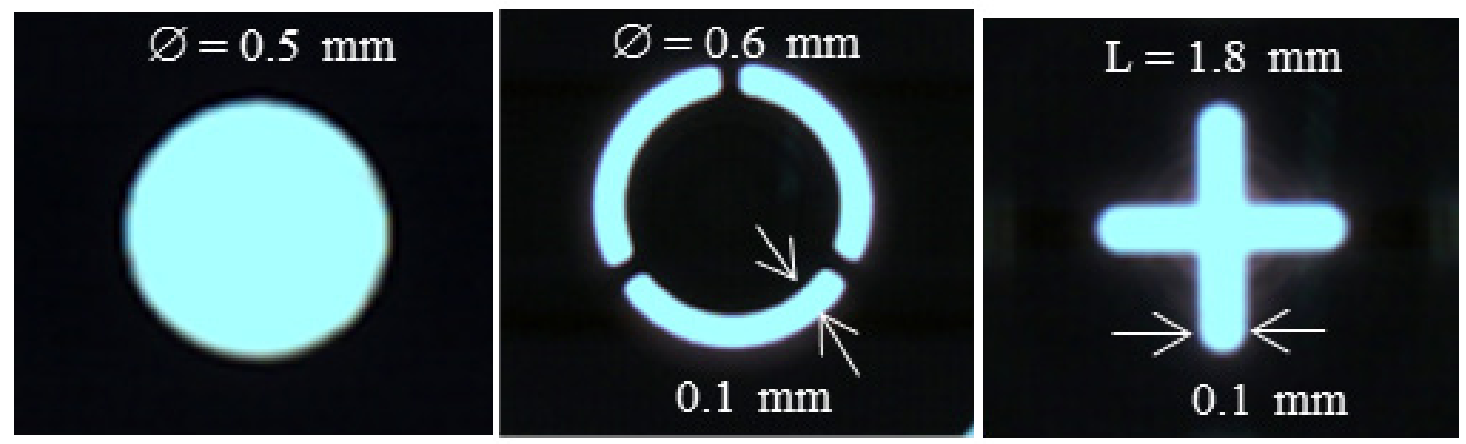

Figure 1. Profiles and dimensions of circular, circular hollow, and cross-shaped spinneret's holes 


\section{Results and discussion}

\subsection{Characterizations of TPP-Mt and TPP-Mt/PP nanocomposites}

Thermogram mass loss curves of the parent Mt and TPPMt were shown in Fig. 2. The parent Mt maintained thermal stability, i.e. it was not thermally decomposed, through the range of temperatures studied. The first mass loss, observed at temperature below $200^{\circ} \mathrm{C}$, was due to the loss of interlayer water. $^{[15]}$ The obtained TPP-Mt exhibited high thermal stability that decomposition occurred at temperature range of 418$576^{\circ} \mathrm{C}$. Such high thermal stability of TPP-Mt was expected to improve the thermal property of TPP-Mt/PP nanocomposites and thus, the flame retardant performance of the resulting nonwovens.

X-ray diffraction obtained from purified Mt and TPP-Mt are shown in Fig. 3. Their diffraction patterns and intensities were comparable, only the peak positions were shifted. The parent Mt exhibited montmorillonite characteristic peak at $2 \theta=5.8^{\circ}$, corresponding basal spacing of $1.53 \mathrm{~nm}$. As the tetraphenyl phosphonium cations were exchanged in Mt interlayer, the basal spacing of TPP-Mt increased to $1.79 \mathrm{~nm}$, indicated by a shift of diffraction peak to a lower $2 \theta=4.9^{\circ}$. The XRD results confirmed the incorporation of TPP into layers of organoclay, which could enhance mixing of TPP-Mt in the PP matrix.

Thermal properties of (1-3\%wt) TPP-Mt/PP nanocomposites, in comparison with $\mathrm{PP}$, were characterized by the TGA analysis at temperature range of $0-600^{\circ} \mathrm{C}$, heating rate $10^{\circ} \mathrm{C} / \mathrm{min}$ under $\mathrm{N}_{2}$ flow of $20 \mathrm{ml} / \mathrm{min}$. Mass loss curves of PP and TPP-Mt/PP nanocomposites are shown in Fig. 4 , and the temperatures at which 5 and 10 percent mass loss $\left(T_{5}\right.$ and $\left.T_{10}\right)$ and maximum rate of mass loss $\left(T_{\max }\right)$ occurred are summarized in Table 1. From the results, TPP-Mt/PP nanocomposites exhibited slightly higher thermal stability than PP such that thermal degradation gradually occurred at slightly higher temperatures $\left(410-420^{\circ} \mathrm{C}\right.$ vs. $403^{\circ} \mathrm{C}$ at $\mathrm{T}_{5}$, and $473-482^{\circ} \mathrm{C}$ vs. $468^{\circ} \mathrm{C}$ at $\mathrm{T}_{\max }$, respectively). The nanocomposites of $2 \% w t$ and $3 \%$ wt TPP-Mt possessed comparable thermal stabilities. In preparing nonwovens for the study on flame retardant property, fibers of $3 \%$ wt TPP-Mt/PP were selected.

Similar observation was reported by $\mathrm{Du}$ et al. ${ }^{[11]}$ that the incorporation of $1.8 \% \mathrm{wt}$. organo-bentonite into intumescent flame retardant polypropylene (PP/IFR) enhanced the thermal

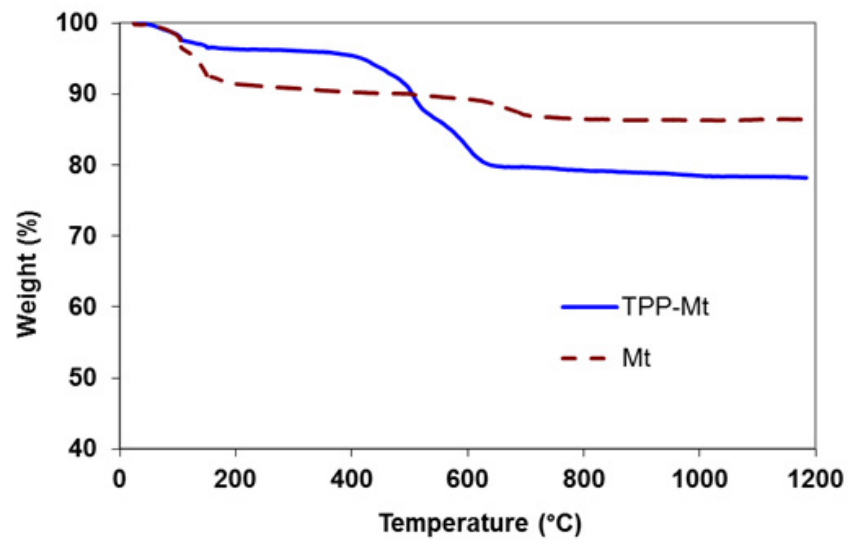

Figure 2. TGA curves of parent Mt and TPP-Mt

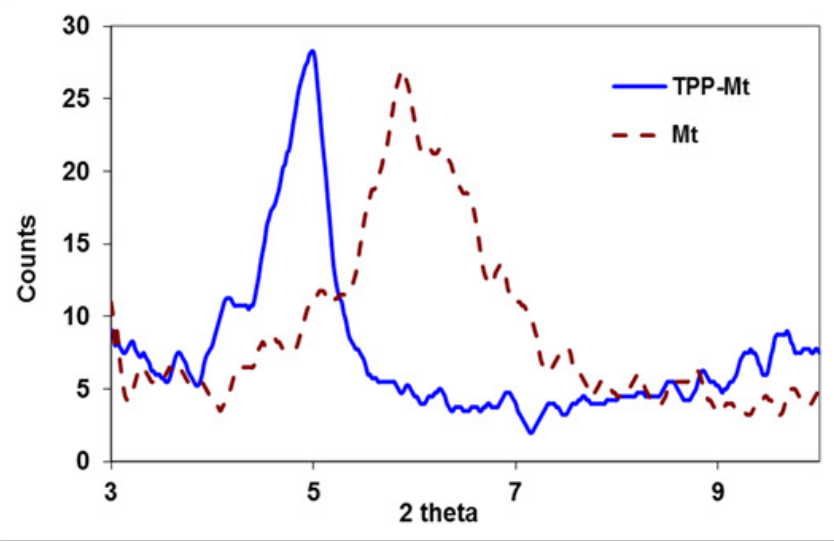

Figure 3. X-ray diffraction of parent Mt and TPP-Mt $\left(2 \theta=3-10^{\circ}\right)$

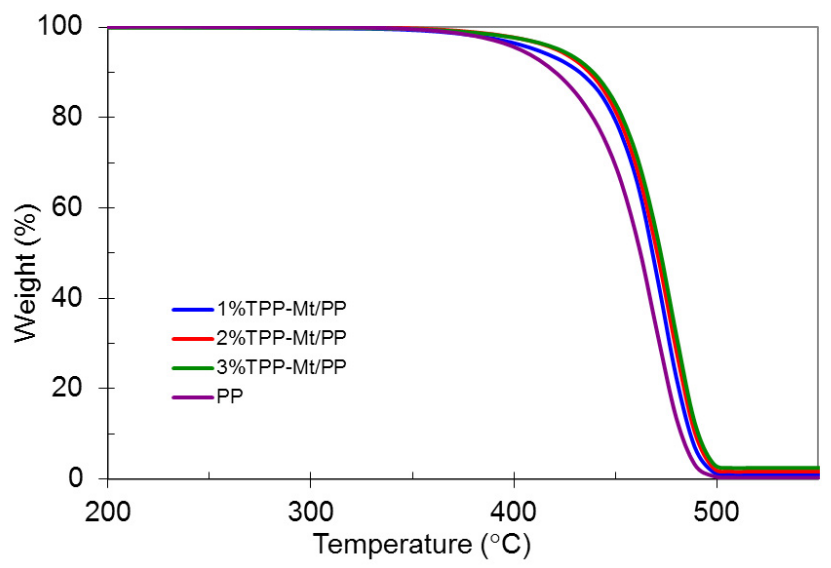

Figure 4. TGA curves of PP and TPP-Mt/PP nanocomposites

Table 1. Decomposition temperatures at $T_{5}, T_{10}, T_{\max }$ of PP and TPP-Mt/PP nanocomposites

\begin{tabular}{|c|c|c|c|}
\hline \multirow{2}{*}{ Sample } & \multicolumn{3}{|c|}{ Decomposition temperature $\left({ }^{\circ} \mathbf{C}\right)$} \\
\cline { 2 - 4 } & $\begin{array}{c}\mathbf{5 \%} \text { mass loss } \\
\left(\mathbf{T}_{5}\right)\end{array}$ & $\begin{array}{c}\mathbf{1 0 \%} \text { mass loss } \\
\left(\mathbf{T}_{10}\right)\end{array}$ & $\begin{array}{c}\text { maximum rate of mass } \\
\text { loss }\left(\mathbf{T}_{\text {max }}\right)\end{array}$ \\
\hline PP & 403.3 & 420.5 & 468.2 \\
\hline $1 \% w t$ TPP-Mt/PP & 410.9 & 432.5 & 472.7 \\
\hline 2\%wt TPP-Mt/PP & 421.2 & 437.2 & 479 \\
\hline 3\%wt TPP-Mt/PP & 422.6 & 439 & 482 \\
\hline
\end{tabular}


stability of nanocomposites such that their maximum weight loss rate temperatures $\left(T_{\max }\right)$ were higher than that of pure PP $\left(343-352^{\circ} \mathrm{C}\right.$ vs. $\left.309^{\circ} \mathrm{C}\right)$. The addition of organo-bentonite into PP/IFR not only delayed the initial decomposition temperature, but also caused an additional thermal stabilization effect on the flame retarded PP.

\subsection{Characterizations of TPP-Mt/PP fibers}

Circular-, circular hollow-, and cross-shaped PP fibers and TPPMt/PP fibers containing 1, 2, and 3\%wt TPP-Mt were prepared and characterized. In fiber spinning, the content of TPP-Mt was limited up to $3 \%$ wt in order to spin fiber continuously without fiber breakage. Aggregation of the TPP-Mt in PP matrix, as well as complex shape of nozzle hole, affected the spinnability of the TPP-Mt/PP fibers. When polymer melt passed through such complex holes, especially with the presence of TPP-Mt, high stress could be developed on the polymer melt and the fiber spinning line was prone to breakage. The obtained PP and TPP-Mt/PP fibers were observed under SEM as shown in Fig. 5. Surface of pure PP fiber was smooth, and became rougher with increasing amount of TPP-Mt due to aggregation of TPP-Mt particles.
In 3\%wt TPP-Mt/PP fibers, the aggregation of TPP-Mt particles were clearly observed. Their sizes were in a range of 6.5-10.5 microns, which were considerably large, and could cause fiber breakage when the fibers were collected at higher speed.

Fiber perimeters of circular-, circular hollow- and cross-shaped TPP-Mt/PP fibers were determined, using the OM equipped with ImageJ software, the results of the same are shown in Fig. 6. Overall, the cross-shaped TPP-Mt/PP fibers showed higher fiber perimeter, that is the larger surface area, than circular and circular hollow-shaped fibers. Such larger surface area allows more possibility of TPP-Mt being exposed to the flame and acted as flame retardant agent. The flame retardant performance of the nonwovens could thus be enhanced.

\subsection{Flame retardant properties of nonwovens}

Dry-laid nonwoven samples of PP and 3wt\% TPP-Mt/PP fibers were fabricated and tested for their flame retardance (ASTM D1230). The sample was held in touch with the flame for 60 seconds before the fire source was removed. The flame propagation on nonwoven sample were recorded at time 0 (first touch), 1, 15, 30, 45 and 60 second.
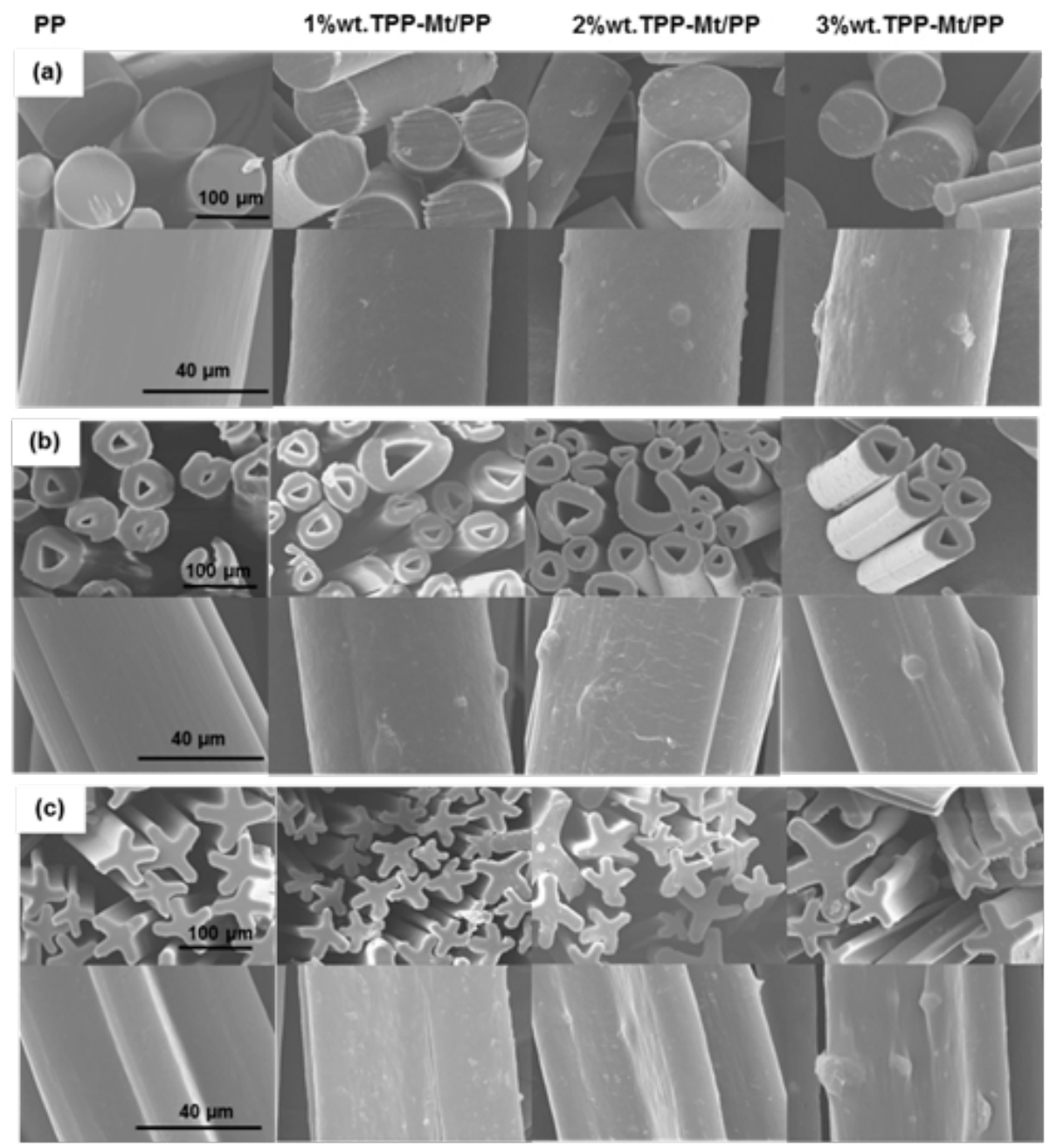

Figure 5. Surface and cross-sectional features of pure PP, (1, 2, and 3\%wt.) TPP-Mt/PP fibers (a) circular-, (b) circular hollow-, (c) cross-shape. 


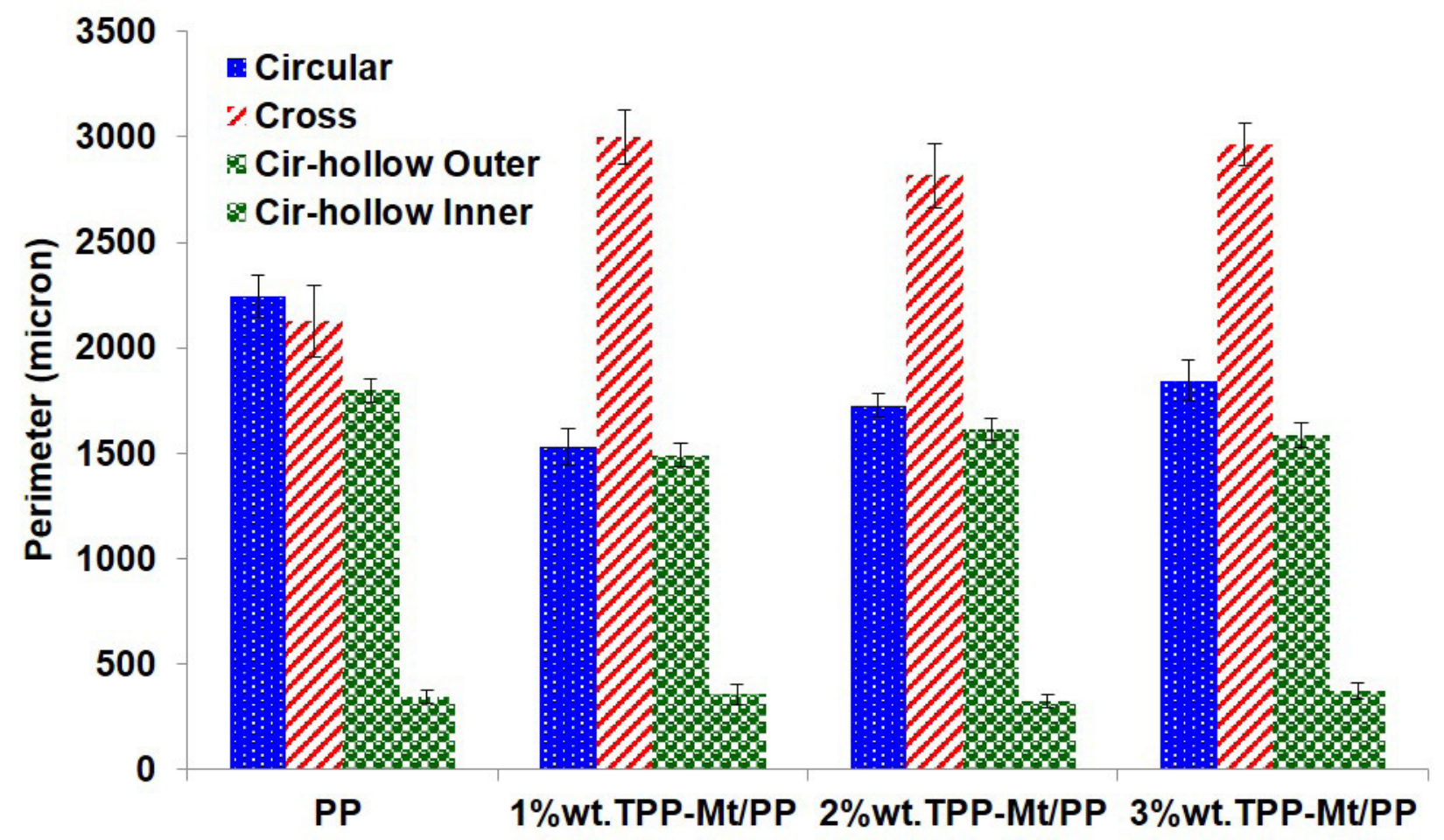

Figure 6. Perimeters of PP and TPP-Mt/PP fibers

The area of burning was determined, using ImageJ software and summarized in Table 2. In testing, the nonwoven samples of circular-shaped PP fibers were quickly burned once they touched the flame. Smoke and polymer melt drips were observed during burning, and the averaged burned area was 91.6 percent (Fig. 7a). The nonwovens of cross-shaped PP fibers, however, showed a tendency of self-extinguishing characteristic that the flame propagation could be stopped after burning (Fig. 7b), and the burned area was 75.9 percent. The nonwovens of TPP-Mt/PP fibers burned more slowly, compared to those of PP fibers, and they exhibited self-extinguishing characteristic such that no polymer melt drip was observed (Fig. 7c-e). Their determined areas of burning were in the range of 14.5-31.6 percent (Table 2).

Table 2. Burned areas in PP and TPP-Mt/PP nonwovens after flame retardant test

\begin{tabular}{|c|c|}
\hline Nonwovens & Ave. Burned area (\%) \\
\hline Circular PP & $91.56 \pm 8.44$ \\
\hline Cross PP & $75.90 \pm 8.89$ \\
\hline Circular 3\%wt TPP-Mt/PP & $31.59 \pm 10.01$ \\
\hline Circular hollow 3\%wt TPP-Mt/PP & $20.05 \pm 7.21$ \\
\hline Cross 3\%wt TPP-Mt/PP & $14.46 \pm 5.42$ \\
\hline
\end{tabular}

The nonwovens of cross-shaped TPP-Mt/PP fibers showed the best flame retardant property, followed by those of circular hollow and circular shapes. This implied that fiber shape was one of the factors that could play roles on flame retardant property. The cross-shaped TPP-Mt/PP fibers possessed grooves between their arms. When they were packed closely into the nonwoven, such grooves could form small channels between the fibers. The formed inter-fiber channels could act as holes to trap the radicals and prevented them from inducing further flame propagation. ${ }^{[25]}$ Likewise, the nonwovens of circular hollow fibers also showed good tendency of self-extinguishing, which was thought to be due to their hollow center that could trap initiating radicals inside, thus reducing flame propagation. Considering the fiber surface area, the extended arms of cross-shaped TPP-Mt/PP fibers yielded larger surface area, compared to circular and circular hollow fibers. Such larger surface area enhanced the possibility that TPP-Mt particles be exposed to the flame, and effectively performed their functions as flame retardant.

\section{Conclusions}

The modified TPP-Mt exhibited high thermal stabilities such that the TGA mass loss due to thermal degradation occurred at temperature range of $418-576^{\circ} \mathrm{C}$. The TPP$\mathrm{Mt} / \mathrm{PP}$ nanocomposites were prepared and melt-spun into shaped fibers (circular, circular hollow, and cross-shaped) containing 1, 2, and 3\%wtTPP-Mt. TGA showed that TPPMt/PP nanocomposites exhibited higher thermal stability than PP such that thermal degradation occurred at a slightly higher temperature $\left(410-420^{\circ} \mathrm{C}\right.$ vs. $403^{\circ} \mathrm{C}$, and $473-482^{\circ} \mathrm{C}$ vs. $468^{\circ} \mathrm{C}$ at $\mathrm{T5}$ and Tmax, respectively). The nanocomposites of $2 \% w t$ and $3 \%$ wt TPP-Mt possessed comparable thermal stabilities. The cross-shaped TPP-Mt/PP fibers showed the largest surface area, and its nonwoven exhibited greater flame retardant property than those of the circular hollow and circular fibers. The nonwovens of circular hollow fibers showed the second best performance in flame retardation. Flame retardant 
(a)

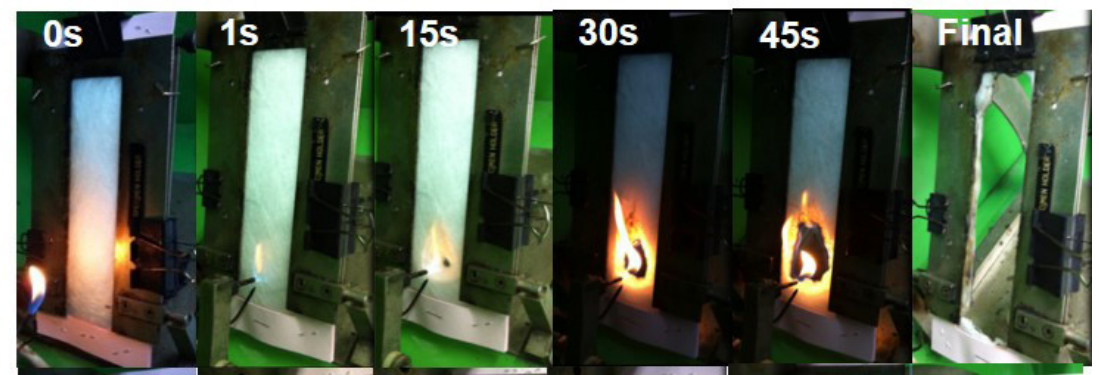

(b)

(c)

(d)

(e)

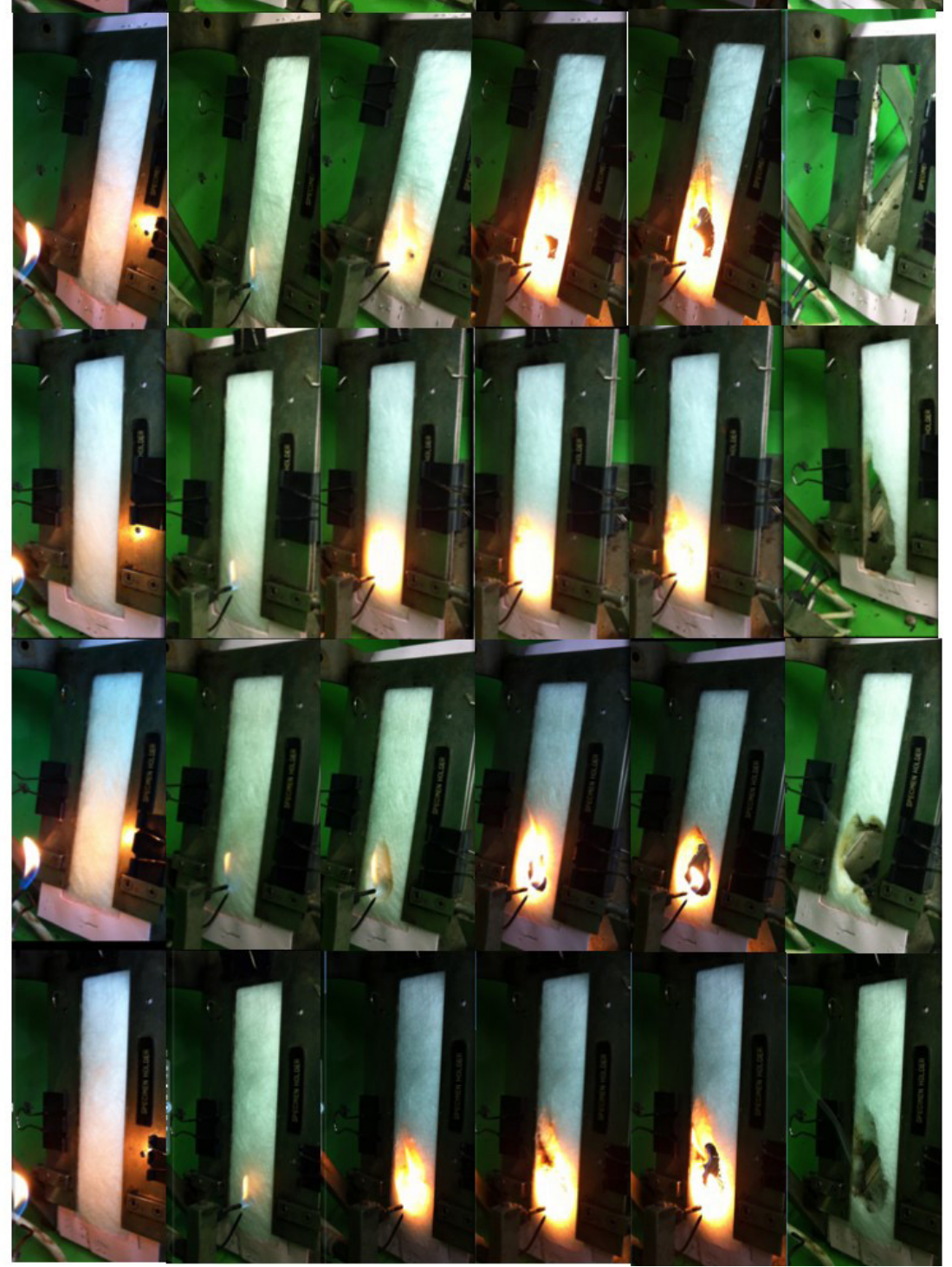

Figure 7. Flame retardant test on nonwovens of (a) circular (b) cross PP fibers, and $3 \%$ wt TPP-Mt/PP fibers of different crosssectional shapes (c) circular (d) circular hollow (e) cross

property observed in nonwovens of TPP-Mt/PP fibers was contributed from both TPP-modified organoclay and fiber shape.

\section{ACKNOWLEDGEMENTS}

This work was supported by the National Science and Technology Development Agency, Thailand (grant number: MT-B53-POL-07-488-I). Our gratitude to HMC polymers for their support on polypropylene resins.

\section{References}

[1] Kotal, M. \& Bhowmick, A.K. (2015). Polymer nanocomposites from modified clays: Recent advances and challenges.Progress in Polymer Science, 51, 127187.

[2] Shah, K.J., Shukla, A.D., Shah, D.O. \& Imae T. (2016). Effect of organic modifiers on dispersion of organoclay in polymer nanocomposites to improve mechanical properties. Polymer, 97, 525-532.

[3] Garcia-Lopez, D., Fernandez, J.F., Merino, J.C. \& Pastor, J.M. (2013). Influence of organic modifier characteristics on 
the mechanical properties of polyamide6/organo-sepiolite nanocomposites. Composites: Part B, 45, 459-465.

[4] Feng, J., Hao, J., Du, J. \& Yang, R. (2012). Effects of organoclay modifiers on the flammability, thermal and mechanical properties of polycarbonate nanocomposites filled with a phosphate and organoclays. Polymer Degradation and Stability, 97, 108-117.

[5] Leszczynska, A., Njuguna, J., Pielichowski, K. \& Banerjee, J.R. (2007). Polymer/montmorillonite nanocomposites with improved thermal properties II. Thermal stability of montmorillonite nanocomposites based on different polymeric matrixes. Thermochimica, 454, 1-22.

[6] Calderon, J.U., Lennox, B. \& Kamal, M.R. (2008). Thermally stable phosphonium-montmorillonite organoclay. Applied Clay Science, 40, 90-98.

[7] Abdallah, W. \& Yilmazer, U. (2011). Novel thermally stable organo-montmorillonites from phosphonium and imidazolium surfactants. Thermochimica Acta, 525, 129140.

[8] Mittal, V. (2012). Modification of montmorillonites with thermally stable phosphonium cations and comparison with alkylammonium montmorillonites. Applied Clay Science, 56:103-109.

[9] Patel, H. A., Somani, R.S., Bajaj, H. C. \& Jasra, R. V. (2007). Preparation and characterization of phosphonium montmorillonite with enhanced thermal stability. Applied Clay Science, 35, 194-200.

[10] Xie, W., Xie, R., Pan, W.P., Hunter, D., Koene, B., Tan, L.S. \& Vaia, R. (2002). Thermal stability of quarternary phosphonium modified montmorillonites. Chemistry of Materials, 14, 4837-4845.

[11] Du, B., Guo, Z., Song, P., Liu, H., Fang, Z. \& Wu, Y. (2009). Flame retardant mechanism of organo-bentonite in polypropylene. Applied Clay Science, 45, 178-184.

[12] Yourdkhani, M., Mousavand, T., Chapleau, N. \& Hubert, P. (2013). Thermal, oxygen barrier and mechanical properties of polylactide-organoclay nanocomposites. Composites Science and Technology, 82,47-53.

[13] Hwang, S., Liu, S., Hsu, P.P, Yeh, J., Yang, J., Chang, K. \& Chu, S. (2011). Effect of organoclay and preparation methods on the mechanical/thermal properties of microcellular injection molded polyamide 6-clay nanocomposites. International Communications in Heat and Mass Transfer, 38, 1219-1225.

[14] Laoutid, F., Persenaire, O., Bonnaud, L. \& Dubois, P. (2013). Flame retardant polypropylene through the joint action of sepiolite and polyamide 6. Polymer Degradation and Stability. 98, 1972-1980.
15] Onder, E., Sarier, N. \& Ersoy, M. S. (2012). The manufacturing of polyamide-and polypropylene-organoclay nanocomposite filaments and their suitability for textile applications. Thermochimica Acta, 543, 37-58.

[16] Bueno, M. A., Aneja, A. P. \& Renner, M. (2004). Influence of the shape of fiber cross section on fabric surface characteristics. Journal of Materials Science, 39, 557-564.

[17] Karaca, E., Ozcelik, F. (2007). Influence of the crosssectional shape on the structure and properties of polyester fibers. Journal of Applied Polymer Science, 103, 2615-2621.

[18] Oh, T. H. (2006). Studies on melt spining process of hollow polyethylene terephthalate fibers. Polymer Engineering and Science, 46, 609-616.

[19] Jung, I., Kim, S. \& Oh, T. H. (2010). Effects of spinning conditions on shape changes of trilobal-shaped fibers. Textile Research Journal, 80, 12-18.

[20] Zhou, J., Li, J., Yu, W., Lin, X. \& Zhou, C. (2010). Studies on the melt spinning process of noncircular fiber by numerical and experimental methods. Polymer Engineering and Science, 50, 1935-1944.

[21] Takarada, W., Ito, H., Kikutani, T. \& Okui, N. (2001). Studies on high-speed melt spinning of noncircular crosssection fibers II. On-line measurement of the spin line, including change in cross-sectional shape. Journal of Applied Polymer Science, 80, 1582-1588.

[22] Yao, D. (2006). Fundamental study of the driving mechanisms for cross-section shape change in highly noncircular fiber spinning. The Fiber Society, Fall Annual Meeting and Technical Conference, 55-56.

[23] Thuc, C.N.H., Grillet, A.C., Reinert, L. \& Ohashi, F. (2010). Separation and purification of montmorillonite and polyethylene oxide modified montmorillonite from Vietnamese bentonites. Applied Clay Science, 49, 229238.

[24] Prahsarn, C., Roungpaisan, N., Suwannamek, N., Klinsukhon, W., Hayashi, H., Kawasaki, K. \& Ebina, T. (2014). Influence of molecular structure of quarternary phosphonium salts on Thai bentonite intercalation. Clays and Clay Minerals, 62(1), 13-19.

[25] Zhang, S. \& Horrocks, A.R. (2003). A review of flame retardant polypropylene fibres. Progress in Polymer Science, 28, 1517-1538. 